\title{
Incoherent imaging of crystals using thermally scattered electrons
}

\author{
BY D. E. JESSON AND S. J. PENNYCOOK \\ Solid State Division, Oak Ridge National Laboratory, Oak Ridge, \\ TN 37831-6030, U.S.A.
}

\begin{abstract}
Thermal diffuse scattering of electrons through large angles by a simple low-index crystal projection is examined in the context of a phonon model, based on the Warren approximation of X-ray diffraction. The scattering from an individual atomic column is visualized in terms of an assembly of independent 'packets' of atoms. Within a packet, the scattering is partially coherent, causing the columnar intensity to deviate from that calculated with an Einstein independent oscillator model. For typical atomic spacings, this deviation is limited to within $20 \%$.
\end{abstract}

\section{Introduction}

It is now over 20 years since the first images of single atoms and atom clusters were obtained in a scanning transmission electron microscope (STEM) equipped with an annular dark-field (ADF) detector (Crewe 1970; Crewe \& Wall 1970; Wall et al. 1974). Such early images of very thin objects were interpreted in the framework of an incoherent particle scattering model, even though they were formed predominantly from coherently scattered electrons, which led naturally to the question of the degree to which the images could be interpreted using incoherent scattering theory (Cowley 1976; Ade 1977; Colliex \& Mory 1983). In a previous paper (Jesson \& Pennycook 1993), it was demonstrated that, for the range of ADF detector angles used in forming these early images, interpretation based on incoherent scattering theory was justified, but with the important proviso that atoms need to be separated in a plane perpendicular to the optic axis.

Interference effects between atoms possessing the same projected coordinates along the axis are preserved in the high-angle signal and must be considered explicitly. This is conveniently illustrated by considering the image of a crystal aligned along a zone axis direction. In the thin crystal limit, the intensity of a column will increase as the number of atoms in the column squared $\left(n^{2}\right)$ and not $n$ as would be expected from incoherent imaging theory. This has led, for example, to a reevaluation (Jesson \& Pennycook 1993) of the early images of a uranium microcrystal (Isaacson et al. 1979).

From the above discussion, it would appear that interference effects along a column are, at least in practice, an inescapable feature of STEM ADF imaging. However, Howie (1979) appreciated that by increasing the inner angle of the ADF detector, it would be possible to suppress Bragg reflections and form images from thermal diffuse scattering (TDS). Images formed in this way are generally referred to as atomic number or Z-contrast images. From a simplified Einstein picture of

Proc. R. Soc. Lond. A (1995) 449, 273-293

Printed in Great Britain
(C) 1995 The Royal Society

273
TEX Paper 
atoms acting as independent oscillators, it can be expected that coherence effects, even along an atomic column, will be destroyed as long as Bragg reflections do not make a significant contribution to the high-angle signal. However, unlike a full phonon TDS description, involving correlated displacements, the Einstein model assumes incoherent scattering from each atom in the vibrating column, and therefore does not properly describe the interference effects present in large-angle TDS. At present, it therefore remains an open question as to the efficiency of phonons in breaking the coherence along an atom column. This issue of longitudinal coherence along a column has recently assumed considerable importance through the development of atomic-resolution Z-contrast imaging techniques where it has now become possible to image columns individually (Pennycook \& Jesson 1990, 1991, 1992). Clearly, atomic correlations along a column could significantly influence the large-angle scattering to the high-angle ADF detector and potentially complicate the simple dependence on columnar scattering cross section as derived from the Einstein theory.

The purpose of this paper is to address the issue of coherence along an atomic column and evaluate the contributions of correlated vibrations to the Z-contrast image. Our approach is based on the approximation of Warren (1990). Although simple in nature, this approach shows clearly the extent to which phonons break the coherence along a column. It includes all orders of phonon scattering and reduces to the coherent and Einstein expressions in the limits of completely coherent and incoherent scattering, respectively.

\section{An intensity expression for Z-contrast imaging}

In this section, we will derive an approximate intensity expression describing Z-contrast imaging. For simplicity, our treatment will retain only the important physics governing the dynamical diffraction of the incident probe with the emphasis placed on a realistic description of atomic vibrations.

A schematic representation of the Z-contrast imaging geometry is given in figure 1 where a coherent probe of atomic dimensions is focused on the top surface of a thin crystal. It is now well established (Fertig \& Rose 1981; Loane et al. 1988) that if the crystal is aligned along a low-index zone axis, the projected atomic columns behave as microlenses, focusing the probe to peak up at the atom sites as shown schematically in figure $2 a$. Interestingly, the peaking of the wave function at the atomic columns closely adheres to the envelope of the surface probe amplitude. This can be explained quantum mechanically by the selection of non-dispersive s-type Bloch states of the fast electron (figure $2 b$ ) over the angular range of the incident probe (Pennycook \& Jesson 1990). We therefore model the probe wave function as single or, where appropriate, clusters of axial s-states weighted by the surface probe, which is known to provide a good description of the current density propagating down the atomic columns underneath the incident probe (Pennycook \& Jesson 1990, 1991). In very thin crystals, s-states are not well resolved from other Bloch states, but they rapidly dominate at the atom sites in the $c a .20 \mathrm{~nm}$ thickness range relevant to Z-contrast imaging. This is shown clearly in figure $2 c$. Note that the scale of interference oscillations between Bloch states in the full calculation is approximately $20 \mathrm{~nm}$, which we shall find is considerably greater than the range of atomic correlations along a column (see $\S 5 b)$. It is, therefore, possible to adopt an s-state model of probe propagation 


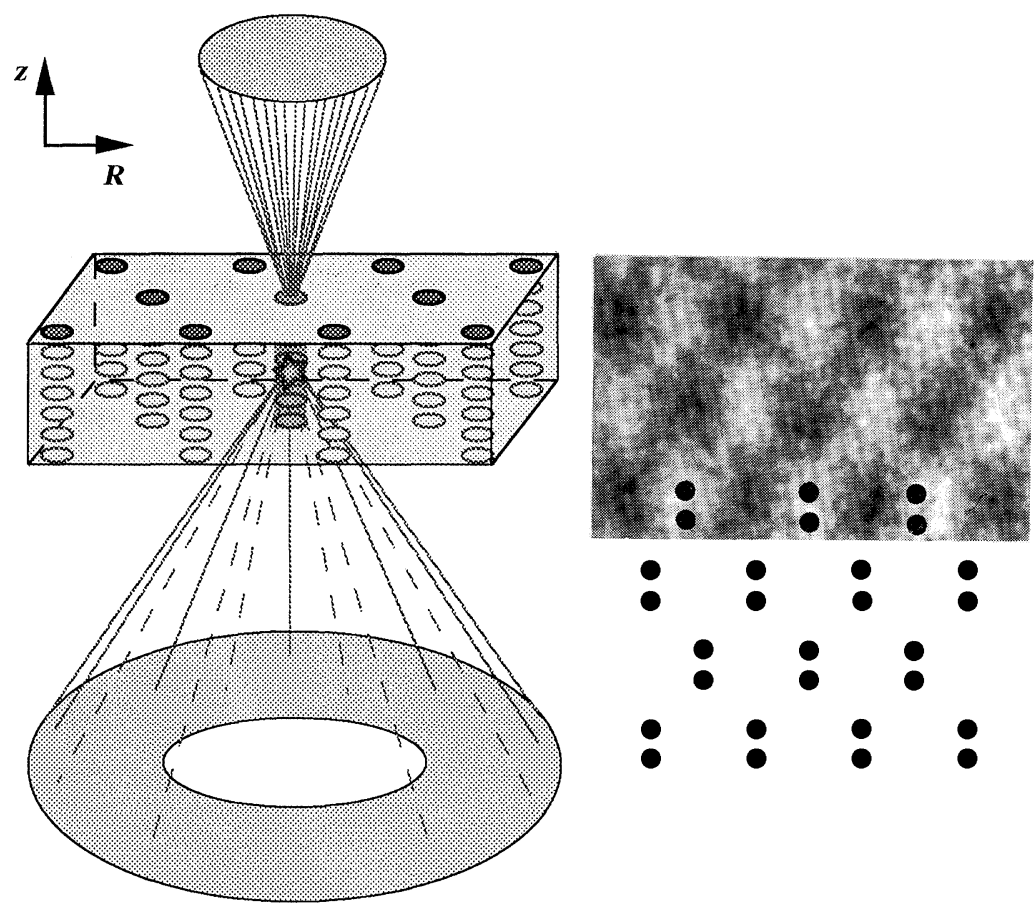

Figure 1. A schematic illustration of the Z-contrast imaging geometry. A focused electron probe channels along the projected atom columns and undergoes a large-angle scattering event to the high-angle detector. Images are formed by scanning the electron probe across the surface and can be interpreted in terms of the projected specimen scattering power. The $\mathrm{Si}\langle 110\rangle$ example at $100 \mathrm{kV}$ illustrates a case where the individual atomic columns comprising a dumbbell separated by $1.36 \AA$ cannot be resolved by the $2.2 \AA$ FWHM probe.

while still retaining a physically reasonable description of correlations along a column.

Weak large-angle scattering from the zero-layer s-states to the high-angle ADF detector is essentially a kinematic process which can be treated using perturbation theory in a distorted-wave Born approximation (see, for example, Schiff 1968; Vincent et al. 1984). Of considerable interest for further simplification is the extent to which the scattering events are localized within the crystal. The largeangle signal can be visualized in terms of three basic components; residual zero layer diffraction, diffraction to higher-order Laue zones (HOLZs) of the reciprocal lattice, and TDS. Large-angle kinematic scattering implicates the sharply varying regions of the atomic potentials. As seen by the s-states, these 'point' (ca. $0.1 \AA$ ) scattering centres are thermally smeared by atomic vibrations. However, typical vibration amplitudes are only of the order of $0.1 \AA$, considerably less than the spatial variation of s-states. This degree of spatial localization about the projected atom sites enables us to write out a simplified expression for residual zero-layer diffraction, HOLZ scattering, and TDS integrated over the high-angle detector as

$$
\begin{aligned}
& I^{\mathrm{H}}\left(\boldsymbol{R}_{0}\right)=\epsilon^{1 \mathrm{~s}^{2}} \sum_{m, n} \tau^{\mathrm{ls}}\left(\boldsymbol{R}_{m}\right) \tau^{\mathrm{ls}}\left(\boldsymbol{R}_{n}\right) P\left(\boldsymbol{R}_{m}-\boldsymbol{R}_{0}\right) P^{*}\left(\boldsymbol{R}_{n}-\boldsymbol{R}_{0}\right) \\
& \times \int_{\text {detector }}^{m, n} f^{2}\left(\boldsymbol{s}^{\mathrm{eff}}\right) \exp \left[\mathrm{i} \boldsymbol{s}^{\mathrm{eff}} \cdot\left(\boldsymbol{r}_{m}-\boldsymbol{r}_{n}\right)\right]\left\langle\exp \left[\mathrm{i} \boldsymbol{s}^{\mathrm{eff}} \cdot\left(\boldsymbol{u}_{m}-\left(\boldsymbol{u}_{n}\right)\right]\right\rangle \mathrm{d} \boldsymbol{s},\right.
\end{aligned}
$$

in which we assume a monatomic crystal containing atoms of scattering factor 


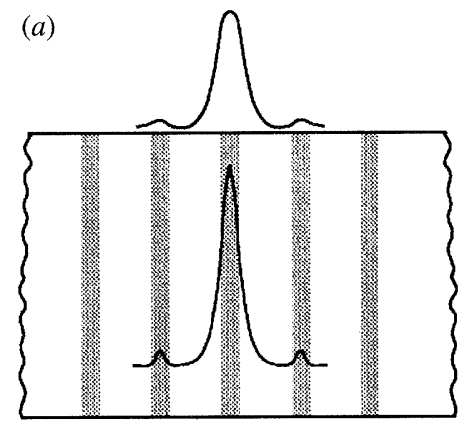

(b)

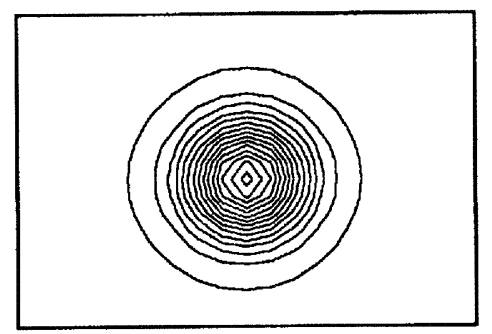

(c)

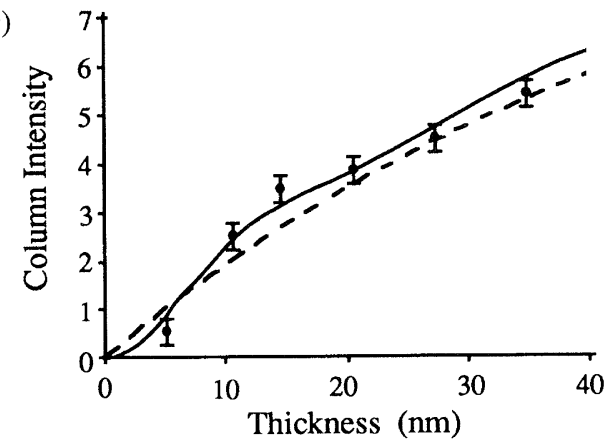

Figure 2. A schematic representation of probe channelling along a simple crystal projection. The peaking of the probe at the positions of the atomic columns in $(a)$ results from the preferential selection of s-type Bloch states as shown in plan view in $(b)$. In $(c)$, we display results for the thickness integrated probe wavefunction intensity at the atom sites with a $2.2 \AA$ probe located over a $\operatorname{Si}\langle 110\rangle$ dumbbell as shown in figure 1 . The solid line corresponds to a full dynamical calculation and is in good agreement with the experimental data points (solid circles). The dashed line represents an independent s-state calculation.

$f\left(\boldsymbol{s}^{\mathrm{eff}}\right)$. Here, $\boldsymbol{u}_{m}$ denotes the instantaneous displacement of the $m$ th atom about its lattice position $\boldsymbol{r}_{m}$. It is convenient to partition the coordinate system into vectors $\boldsymbol{z}$ and $\boldsymbol{R}$, which are parallel and perpendicular to the optical axis respectively (see figure 1). In this way, we can specify the position of the $m$ th lattice site $\boldsymbol{r}_{m}=\left\{\boldsymbol{R}_{m}, z_{m}\right\} . P(\boldsymbol{R})$ represents the probe amplitude corrected for channelling, which apart from an overall scaling factor is very similar to the incident probe profile (Pennycook \& Jesson 1991). With the probe located at $\boldsymbol{R}_{0}$, the amplitude illuminating an atom column at $\boldsymbol{R}_{m}$ is $P\left(\boldsymbol{R}_{m}-\boldsymbol{R}_{0}\right)$, which excites an s-state $\tau^{\mathrm{ls}}\left(\boldsymbol{R}_{m}\right)$ of excitation amplitude $\epsilon^{\mathrm{ls}}$. The modified deviation parameter $\boldsymbol{s}^{\mathrm{eff}}$ takes into account the additional deviation introduced by the s-state wavevector. Here and henceforth, we put $s^{\text {eff }}$ equal to the conventional value $s$ without significantly affecting the results of our calculations at large scattering angles. In the further interest of clarity, absorption has not been included explicitly in (2.1). We will, however, introduce the necessary modifications in $\S 6$.

The single s-state model, equation (2.1), will be a good approximation if the thickness integrated ADF signal is dominated by kinematic scattering from a single s-state or s-state cluster. The localized potential approximation will somewhat underestimate the enhancement of the $f(s)$ by s-states and reduce the overall magnitude of large-angle scattering. The model does, however, fully address the three-dimensional nature of the problem within a kinematic approximation and is therefore suitable for the investigation of coherence along an atomic column.

Proc. R. Soc. Lond. A (1995) 
The important atomic correlation information in (2.1) is contained in the time average term $\left\langle\exp \left[i \boldsymbol{s}^{\mathrm{eff}} \cdot\left(\boldsymbol{u}_{m}-\boldsymbol{u}_{n}\right)\right]\right\rangle$. To evaluate this quantity, it is first necessary to make approximations. In $\S 3$, we begin with the analytical approach of Warren (1990), which provides the most satisfactory basis for evaluating the time average. Existing approaches in the literature and their limitations in describing atomic correlations involved in large-angle scattering will be considered in $\S 4$ and $\S 5$.

\section{The Warren approximation}

In this section, we perform the time average in (2.1), based on the treatment given by Warren (1990). The chief advantage of this approach is that through a series of reasonable approximations, the time average can be obtained in terms of a simple tabulated function. Most importantly, the approach retains important information on the atomic correlations contributing to the high-angle signal. It has become customary to evaluate the time average in (2.1) by expressing the atomic displacements in terms of a set of normal modes (see, for example, James 1954):

$$
\boldsymbol{u}_{n}=\sum_{q, j} a_{q j} \hat{\boldsymbol{e}}_{q j} \cos \left\{\omega_{q j} t-2 \pi \boldsymbol{q} \cdot \boldsymbol{r}_{n}-\delta_{q j}\right\}
$$

where $a_{q j}$ is the amplitude of the wave of wavevector $q$ and angular frequency $\omega_{q j}$. The arbitrary phase $\delta_{q j}$ reflects the fact that there is no phase relationship between different waves and $\hat{\boldsymbol{e}}_{q j}$ specifies a unit vector in one of the three independent vibration directions $(j=1,3)$ indicating the polarization of the wave. It is usual to express the time average in terms of the physically meaningful mean square amplitude for each wave (Warren 1990),

$$
\left\langle\exp \left[\mathrm{i} \boldsymbol{s}^{\mathrm{eff}} \cdot\left(\boldsymbol{u}_{m}-\boldsymbol{u}_{n}\right)\right]\right\rangle=\exp \left[\sum_{q, j} G_{q j} \cos \left\{2 \pi \boldsymbol{q} \cdot\left(\boldsymbol{r}_{m}-\boldsymbol{r}_{n}\right)\right\}\right] \exp (-2 M),
$$

where the coefficients

$$
G_{q j}=\frac{1}{2}\left(s \cdot \hat{\boldsymbol{e}}_{q j}\right)^{2}\left\langle a_{q j}^{2}\right\rangle .
$$

Here, $\exp (-M)$ is the usual Debye-Waller factor term with $M=B s^{2}$. Now consider the average energy per elastic wave given by

$$
\left\langle E_{q j}\right\rangle=\frac{1}{2} N m \omega_{q j}^{2}\left\langle a_{q j}^{2}\right\rangle
$$

where there are $N$ atoms of mass $m$ present in the crystal. From (3.3) we have

$$
G_{q j}=\frac{s^{2} \cos ^{2}\left(s, \hat{e}_{q j}\right)}{N m \omega_{q j}^{2}}\left\langle E_{q j}\right\rangle
$$

where $\cos \left(\boldsymbol{s}, \hat{\boldsymbol{e}}_{q j}\right)$ represents the cosine of the angle between vectors $\boldsymbol{s}$ and $\hat{\boldsymbol{e}}_{q j}$. Treating each elastic wave as a harmonic oscillator, then apart from very low temperatures $T$, we have $\left\langle E_{q j}\right\rangle=k T$. With the further assumption of a Debye dispersion relation $\left(\omega_{q j}=2 \pi v q\right)$ in which all waves are assumed to have the same mean velocity $v$, then from (3.5), we obtain

$$
\sum_{j} G_{q j}=\frac{s^{2} k T}{4 \pi^{2} q^{2} N m v^{2}}
$$

Proc. R. Soc. Lond. A (1995) 
278

D. E. Jesson and S. J. Pennycook

Replacing the Brillouin zone boundary at $q_{\mathrm{B}}$ by a sphere of equal volume $\frac{4}{3} \pi q_{\mathrm{B}}^{3}$ and noting $\hbar \omega_{\mathrm{B}}=k \Theta_{\mathrm{D}}\left(\Theta_{\mathrm{D}}\right.$ is the Debye temperature $)$ then $v=k \Theta_{\mathrm{D}} / h q_{\mathrm{B}}$. Writing $s=4 \pi \sin \theta_{\mathrm{B}} / \lambda$ in terms of the Bragg angle $\theta_{\mathrm{B}}$ and incident electron wavelength $\lambda$ and defining

$$
Y_{m n}=\sum_{q, j} G_{q j} \cos \left[2 \pi \boldsymbol{q} \cdot\left(\boldsymbol{r}_{m}-\boldsymbol{r}_{n}\right)\right]
$$

then gives

$$
Y_{m n}=\sum_{q} \frac{4 h^{2} T}{N m k \Theta_{\mathrm{D}}^{2}}\left(\frac{\sin \theta_{\mathrm{B}}}{\lambda}\right)^{2} \frac{q_{\mathrm{B}}^{2}}{q^{2}} \cos \left\{2 \pi \boldsymbol{q} \cdot\left(\boldsymbol{r}_{m}-\boldsymbol{r}_{n}\right)\right\}
$$

from (3.6).

Following Warren (1990) and using the standard result for the Debye-Waller factor,

$$
2 M=\frac{12 h^{2} T}{m k \Theta_{m}^{2}}\left[\Phi(x)+\frac{1}{4} x\right]\left(\frac{\sin \theta_{\mathrm{B}}}{\lambda}\right)^{2}
$$

with the approximations $\left[\Phi(x)+\frac{1}{4} x\right]=1$ and $\Theta_{m}=\Theta_{\mathrm{D}}$, we obtain

$$
Y_{m n}=\frac{2 M}{3 N} q_{\mathrm{B}}^{2} \sum_{q} \frac{\cos \left\{2 \pi \boldsymbol{q} \cdot\left(\boldsymbol{r}_{m}-\boldsymbol{r}_{n}\right)\right\}}{q^{2}} .
$$

The summation over modes is then replaced by an integration in the Brillouin zone using a density of points $N /\left(\frac{4}{3} \pi q_{\mathrm{B}}^{3}\right)$ :

$$
\begin{aligned}
Y_{m n} & =\frac{2 M q_{\mathrm{B}}^{2}}{3 N} \int_{0}^{q_{\mathrm{B}}} \int_{0}^{\pi} \frac{\cos \left\{2 \pi q r_{m n} \cos \phi\right\}}{q^{2}} \frac{N}{\frac{4}{3} \pi q_{\mathrm{B}}^{3}} 2 \pi q^{2} \sin \phi \mathrm{d} \phi \mathrm{d} q \\
& =2 M \frac{\operatorname{Si}\left(2 \pi q_{\mathrm{B}} r_{m n}\right)}{2 \pi q_{\mathrm{B}} r_{m n}} .
\end{aligned}
$$

Here, $r_{m n}=\left|\boldsymbol{r}_{m}-\boldsymbol{r}_{n}\right|$ and $\operatorname{Si}(x)$ is the sine integral function,

$$
\operatorname{Si}(x)=\int_{0}^{x} \frac{\sin u}{u} \mathrm{~d} u .
$$

In the Warren approximation, the time average (equation (3.2)) becomes

$$
\left\langle\exp \left[\mathrm{i} \boldsymbol{s}^{\mathrm{eff}} \cdot\left(\boldsymbol{u}_{m}-\boldsymbol{u}_{n}\right)\right]\right\rangle=\exp \left\{2 M\left[\frac{\operatorname{Si}\left(2 \pi q_{\mathrm{B}} r_{m n}\right)}{2 \pi q_{\mathrm{B}} r_{m n}}-1\right]\right\} .
$$

Inserting (3.13) into (2.1) then provides the intensity expression,

$$
\begin{aligned}
& I^{\mathrm{H}}\left(\boldsymbol{R}_{0}\right)=\epsilon^{\mathrm{ls}^{2}} \sum_{m, n} \tau^{\mathrm{ls}}\left(\boldsymbol{R}_{m}\right) \tau^{\mathrm{ls}^{*}}\left(\boldsymbol{R}_{n}\right) P\left(\boldsymbol{R}_{m}-\boldsymbol{R}_{0}\right) P^{*}\left(\boldsymbol{R}_{n}-\boldsymbol{R}_{0}\right) \\
& \quad \times \int_{\text {detector }} f^{2}(\boldsymbol{s}) \exp \left\{\mathrm{i} \boldsymbol{s} \cdot\left(\boldsymbol{r}_{m}-\boldsymbol{r}_{n}\right)\right\} \exp \left\{2 M\left[\frac{\mathrm{Si}\left(2 \pi q_{\mathrm{B}} r_{m n}\right)}{2 \pi q_{\mathrm{B}} r_{m n}}-1\right]\right\} \mathrm{d} \boldsymbol{s} .
\end{aligned}
$$

This is our basic result describing large-angle electron scattering which will be applied in subsequent sections to discuss atomic correlations in Z-contrast images.

Proc. R. Soc. Lond. A (1995) 
(a)

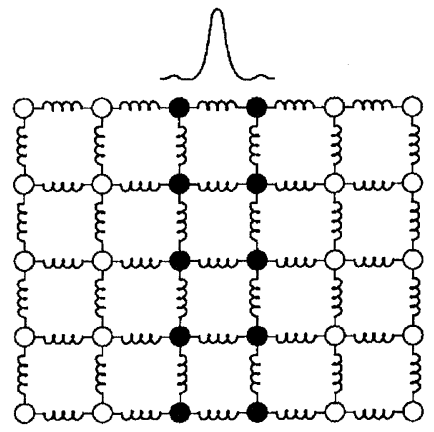

(b)

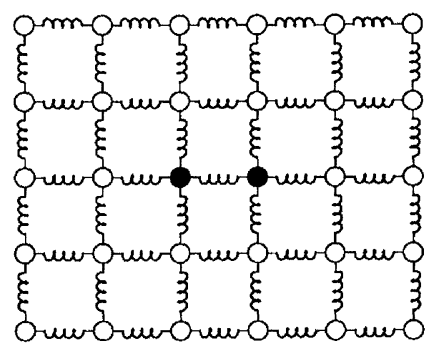

(c)

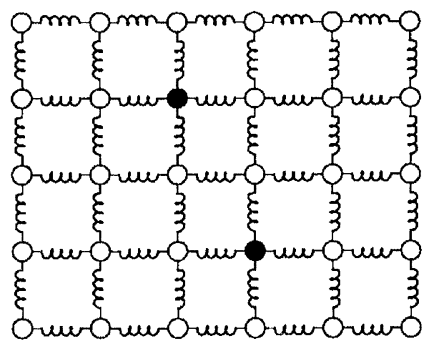

Figure 3. (a) A schematic representation of the surface probe illuminating two atomic columns. Interference between atoms located in different atomic columns $((b)$ and $(c))$ is termed lateral coherence.

\section{Lateral coherence}

In this section, we will apply (3.14) to discuss lateral interference effects present in Z-contrast images of low index crystal projections. In the context of atomicresolution STEM imaging, it is instructive to consider the situation where the incident electron probe illuminates only two neighbouring columns of the crystal as represented schematically in figure $3 a$. We refer to interference effects involving atoms located in different columns (figure $3 b$ or $3 c$ ) as transverse or lateral coherence. Longitudinal coherence involving interferences between atoms in the same column will be considered in $\S 5$.

\section{(a) Stationary atoms}

We begin our discussion of lateral coherence by considering the intensity contribution resulting from the atom pair represented in figure $3 b$ for the specific case of a non-vibrating crystal. This idealized coherent scattering limit can never be reached in practice, but does provide useful insight into the geometrical role of the high-angle detector. The significance of phonons will be considered later 

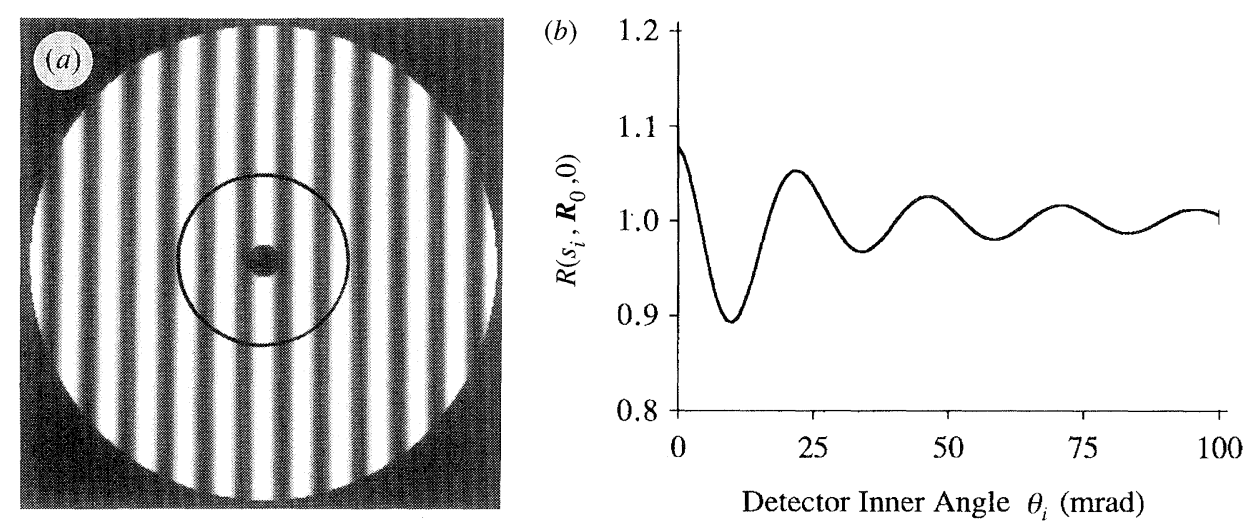

Figure 4. (a) Detector plane intensity distribution resulting from two point scatterers separated by $1.5 \AA$. The inner and outer detector angles are 10.3 and $150 \mathrm{mrad}$, respectively. The solid circle shows that by increasing the inner detector angle to $50 \mathrm{mrad}$, more interference fringes are sampled at the detector periphery. (b) Ratio of the ADF signal to the incoherent signal $\left[\boldsymbol{R}\left(s_{i}, \boldsymbol{R}_{0}, 0\right)\right]$ for two stationary $\mathrm{Si}$ atoms separated by $1.5 \AA$ as a function of inner detector angle. The probe is located between the atom pair.

(a)

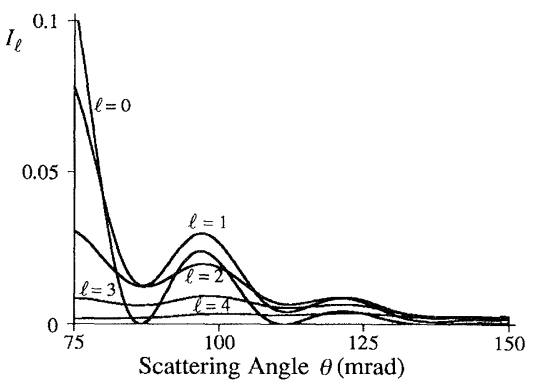

(b)

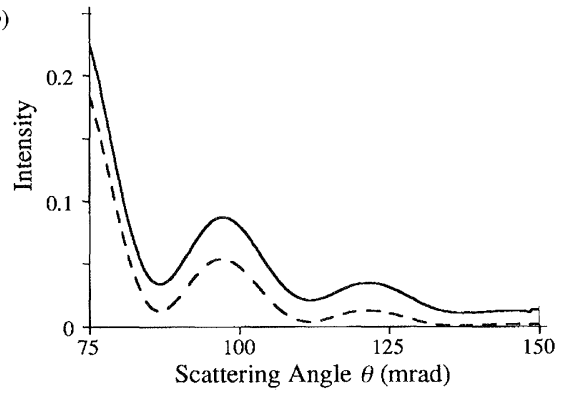

Figure 5. Multiphonon intensity distribution terms $I_{l}$ plotted over the range of the ADF detector for $B=0.45$. (b) Total intensity (equation 4.4) calculated in multiphonon (solid line) and single phonon (dashed line) models. The atom separation is $1.5 \AA$.

in $\S 4 b$. For a non-vibrating simple cubic crystal, the atom pair in figure $3 b$ will contribute an intensity distribution in the ADF detector plane (equation (3.14)),

$$
\begin{aligned}
I\left(\boldsymbol{R}_{0}, \boldsymbol{s}\right)=\epsilon^{1 \mathrm{~s}^{2}} \tau^{1 \mathrm{~s}^{2}}\left(\boldsymbol{R}_{1}\right) f^{2}(\boldsymbol{s})\left[P^{2}\left(\boldsymbol{R}_{1}-\boldsymbol{R}_{0}\right)+P^{2}\left(\boldsymbol{R}_{2}-\boldsymbol{R}_{0}\right)\right. \\
\left.+2 \operatorname{Re}\left\{P\left(\boldsymbol{R}_{1}-\boldsymbol{R}_{0}\right) P^{*}\left(\boldsymbol{R}_{2}-\boldsymbol{R}_{0}\right) \cos [\Delta \boldsymbol{R} \cdot \boldsymbol{s}]\right\}\right],
\end{aligned}
$$

where $\Delta \boldsymbol{R}=\boldsymbol{R}_{1}-\boldsymbol{R}_{2}$ defines the separation between the atoms located at $\left(\boldsymbol{R}_{1}, z_{1}\right)$ and $\left(\boldsymbol{R}_{2}, z_{1}\right)$. Note we have neglected terms in $\sin (\Delta \boldsymbol{R} \cdot \boldsymbol{s})$, which would integrate to zero over the ADF detector.

The properties of (4.1) with regard to incoherent imaging have been considered in detail by Jesson \& Pennycook (1993) (see also Gibson \& Howie 1978). The first two terms describe incoherent scattering from each atom whereas the third term introduces interference fringes into the intensity distribution. This is shown schematically in figure $4 a$ for an atom pair separated by $1.5 \AA$. Here, the atomic scattering factor dependence has been removed from the intensity distribution to emphasize the fringe pattern. The important role of the high-angle detector geometry is clearly evident. Increasing the inner detector angle $\theta_{\mathrm{i}}$ samples 
more fringes around the detector periphery. The third term in (4.1), therefore, decreases, and the signal approaches the result anticipated from incoherent imaging theory (figure $4 b$ ). In real space, increasing $\theta_{\mathrm{i}}$ reduces the lateral coherence length by decreasing the width of the ADF detector function $d(\boldsymbol{R})$. Based on this description, a useful detector geometry criterion for the incoherent imaging of two point scatterers separated by a distance $\Delta R$ was given by Jesson \& Pennycook (1993) as

$$
\theta_{\mathrm{i}}^{\mathrm{inc}}=1.22 \lambda / \Delta R,
$$

where $\lambda$ is the incident electron wavelength. For $100 \mathrm{keV}$ electrons and $\Delta R=$ $1.5 \AA$, this gives $\theta_{i}^{\text {inc }}=30 \mathrm{mrad}$, which, from figure $4 b$, indicates that the deviation from the incoherent signal is less than $5 \%$.

The high-angle detector, therefore, efficiently destroys interference effects between atoms located in different atomic columns, even in the limit of nonvibrating stationary atoms. Thermal vibrations can only assist in this process, and this will be considered in the following section.

\section{(b) Vibrating atoms}

We introduce phonons by expanding the time average (equation (3.13)) in powers of the sine integral function,

$$
\begin{aligned}
\left\langle\operatorname { e x p } \left[\mathrm { i } \boldsymbol { s } \cdot \left(\boldsymbol{u}_{m}-\right.\right.\right. & \left.\left.\left.\boldsymbol{u}_{n}\right)\right]\right\rangle=\exp (-2 M)\left\{1+2 M \frac{\operatorname{Si}\left(2 \pi q_{\mathrm{B}} r_{m n}\right)}{2 \pi q_{\mathrm{B}} r_{m n}}\right. \\
+ & \left.\frac{(2 M)^{2}}{2 !}\left[\frac{\operatorname{Si}\left(2 \pi q_{\mathrm{B}} r_{m n}\right)}{2 \pi q_{\mathrm{B}} r_{m n}}\right]^{2}+\cdots+\frac{(2 M)^{l}}{l !}\left[\frac{\operatorname{Si}\left(2 \pi q_{\mathrm{B}} r_{m n}\right)}{2 \pi q_{\mathrm{B}} r_{m n}}\right]^{l}\right\} .
\end{aligned}
$$

Inserting the expansion into the intensity expression (2.1) then produces a series of intensity terms $I_{l}$. For the pair of atoms embedded in a simple cubic vibrating crystal, as shown in figure $3 b$, we may then write the intensity distribution in the detector plane as

$$
I\left(\boldsymbol{R}_{0}, s\right)=\sum_{l} I_{l}
$$

where

$$
\begin{aligned}
I_{l}=\epsilon^{1 \mathrm{~s}^{2}} \tau^{1 \mathrm{~s}^{2}}\left(\boldsymbol{R}_{1}\right) f^{2}(\boldsymbol{s}) \exp (-2 M) \frac{(2 M)^{l}}{l !}\left[P^{2}\left(\boldsymbol{R}_{1}-\boldsymbol{R}_{0}\right)+P^{2}\left(\boldsymbol{R}_{2}-\boldsymbol{R}_{0}\right)\right. \\
\left.+2 \operatorname{Re}\left\{P\left(\boldsymbol{R}_{1}-\boldsymbol{R}_{0}\right) P^{*}\left(\boldsymbol{R}_{2}-\boldsymbol{R}_{0}\right)\right\}[\operatorname{Si}(\pi) / \pi]^{l} \cos (\Delta \boldsymbol{R} \cdot \boldsymbol{s})\right]
\end{aligned}
$$

The intensity contributions $I_{l}$ are plotted over the range of the ADF detector in figure $5 a$. The zero-order term $(l=0)$ is similar to the expression for coherent scattering from a pair of stationary atoms (equation (4.1)) except it is reduced by the temperature factor $\exp (-2 M)$. Higher-order terms correspond to scattering events which simultaneously involve the creation or annihilation of $l$ phonons. It can be seen that the interference maxima are less pronounced for the higher-order terms and that multiphonon scattering events increase in importance at larger scattering angles. This suggests that it is necessary to include higher-order terms in large-angle TDS models. Recently, however, Wang \& Cowley $(1989,1990)$ have utilized a first-order TDS model, equivalent to truncating the expansion in (4.4) to the $l=1$ term. Although this can be a good approximation at low angles (Takagi 1958), figure $5 b$ shows that a first-order TDS model can significantly underestimate the total scattering in the vicinity of the high-angle detector (see also 


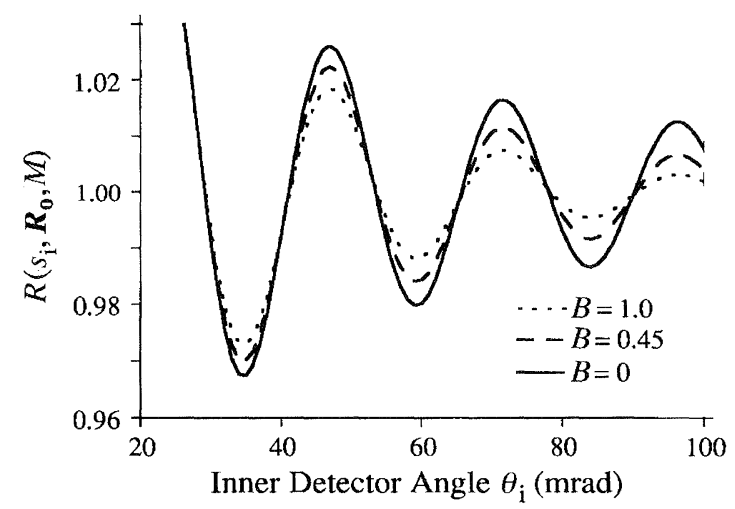

Figure 6. Ratio of the ADF signal to the incoherent signal for two vibrating Si atoms separated by $1.5 \AA$ and embedded in a crystal as in figure $3 b$. The probe is centred exactly between the atom positions. The ratio is plotted as a function of inner detector angle for $B=0,0.45$, and 1.0 .

Hall 1965). Apart from ignoring coherence effects along the column (see $\S 6$ ), the neglect of multiphonon terms by Wang \& Cowley $(1989,1990)$ underestimates the effective TDS scattering cross section per atom in Z-contrast imaging. Consider, for example, a single atom embedded in a vibrating crystal which obeys the Einstein model for thermal vibrations. The ADF intensity resulting from single phonon scattering as a fraction of the total TDS cross section involving a sum over all multiphonon processes is given by

$$
\begin{aligned}
Q_{1} & =I_{1} / \sum_{l} I_{l}-I_{0} \\
& =\int_{s_{\mathrm{i}}}^{s_{\mathrm{o}}} f^{2}(s) \exp (-2 M) 2 M s \mathrm{~d} s / \int_{s_{\mathrm{i}}}^{s_{\circ}} f^{2}(s)[1-\exp (-2 M)] s \mathrm{~d} s,
\end{aligned}
$$

where $s_{\mathrm{i}}$ and $s_{\mathrm{O}}$ correspond to the inner and outer peripheries of the ADF detector. $Q_{1}$ is a factor of 2.6 too small for a $75-150 \mathrm{mrad}$ detector (with $B=0.45$ ). This discrepancy increases at larger scattering angles and/or for greater Debye-Waller factors, explaining why Wang \& Cowley found coherent scattering to contribute significantly to the high-angle signal.

It is clear from (4.5) that thermal vibrations assist in suppressing interference between atoms located in different columns. To quantify this, we consider the ratio of the ADF signal to the ideal incoherent signal for the two vibrating embedded atoms (figure $3 b$ ),

$$
R\left(s_{\mathrm{i}}, \boldsymbol{R}_{0}, M\right)=1+\frac{2 \operatorname{Re}\left[P\left(\boldsymbol{R}_{1}-\boldsymbol{R}_{0}\right) P^{*}\left(\boldsymbol{R}_{2}-\boldsymbol{R}_{0}\right)\right]}{\left[P^{2}\left(\boldsymbol{R}_{1}-\boldsymbol{R}_{0}\right)+P^{2}\left(\boldsymbol{R}_{2}-\boldsymbol{R}_{0}\right)\right]} \xi\left(s_{\mathrm{i}}, \Delta R, M\right),
$$

where

$$
\xi\left(s_{\mathrm{i}}, \Delta R, M\right)=\int_{s_{\mathrm{i}}}^{\infty} f^{2}(s) s J_{0}(s \Delta R) \exp \left\{2 M\left[\frac{\operatorname{Si}(\pi)}{\pi}-1\right]\right\} \mathrm{d} s / \int_{s_{\mathrm{i}}}^{\infty} f^{2}(s) s \mathrm{~d} s .
$$

Equations (4.7) and (4.8) are directly analogous to (31) and (32) of Jesson \& Pennycook (1993) for the case of stationary atoms where $\xi$ was interpreted as the real part of the complex degree of coherence. $R\left(s_{\mathrm{i}}, \boldsymbol{R}_{0}, M\right)$ is plotted in figure 6 as a function of inner detector angle $\theta_{\mathrm{i}}$ and Debye-Waller parameter $B$ for an atom 


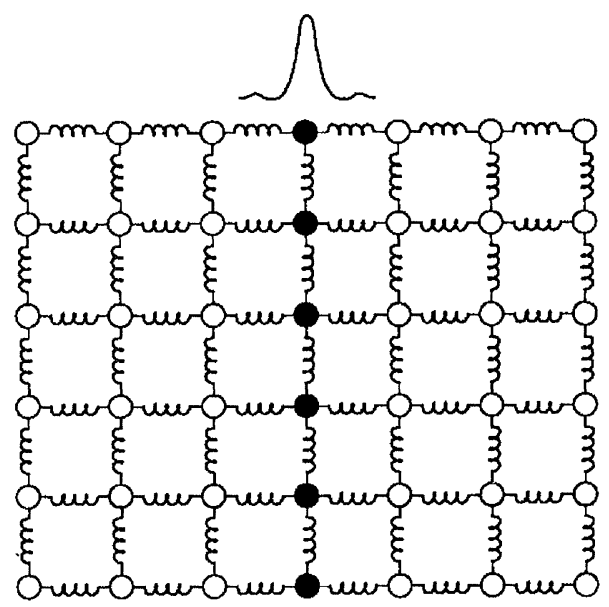

Figure 7. A schematic representation of an individual column (shaded atoms) embedded in a vibrating crystal. Longitudinal coherence involves interference effects between atoms within the column.

separation of $1.5 \AA$. The minimum at around $35 \mathrm{mrad}$ closely corresponds to the stationary atom condition, equation (4.2). At this point, the error in assuming incoherent imaging theory is only $3 \%$. Increasing the amplitude of thermal vibrations further suppresses interference effects, particularly at large scattering angles. However, the geometrical role of the detector still dominates in attaining lateral incoherence between columns at relatively low $\theta_{\mathrm{i}}$. It would, therefore, appear reasonable to utilize (4.2) to define the detector geometry required for lateral incoherence, even in the presence of thermal vibrations.

\section{Longitudinal coherence}

In the previous section, we have demonstrated that the lateral coherence between atoms located in different atomic columns of a low-index crystal projection can be efficiently suppressed by the Z-contrast imaging geometry. In this section, we consider longitudinal coherence in the scattering from atoms in the same atomic column. We assume that the inner detector angle is chosen to meet the criterion for lateral incoherence as discussed in $\S 4$. It is, therefore, sufficient to consider the scattering from an individual column embedded in a vibrating crystal (figure 7). The intensity from such a column, located at $\boldsymbol{R}_{\mathrm{c}}$, is given by (3.14):

$$
\begin{aligned}
I^{\mathrm{H}}\left(\boldsymbol{R}_{0}\right)=\epsilon^{1 \mathrm{~s}^{2}} \tau^{1 \mathrm{~s}^{2}} P^{2}\left(\boldsymbol{R}_{\mathrm{c}}-\boldsymbol{R}_{0}\right) \sum_{m, n} \int_{s_{\mathrm{i}}}^{s_{\circ}} f^{2}(s) \cos \left\{\frac{\pi s^{2}}{\chi} z_{m n}\right\} \\
\times \exp \left\{2 M\left[\frac{\mathrm{Si}[(m-n) \pi]}{|(m-n) \pi|}-1\right]\right\} 2 \pi s \mathrm{~d} s,
\end{aligned}
$$

where the summation is performed over atoms within the column and $z_{m n}=$ $z_{m}-z_{n}$. Here, $\chi$ is the magnitude of the incident electron wavevector. The properties of (5.1) are illustrated in figure $8 a-d$ where azimuthally integrated intensity distributions (i.e. integrated over a narrow annulus at $\theta$ ) corresponding to columns of length $2,4,18$ and $200 \AA$ are plotted in the range of the ADF detector. In each case, this is compared with the intensity distribution associated with an 

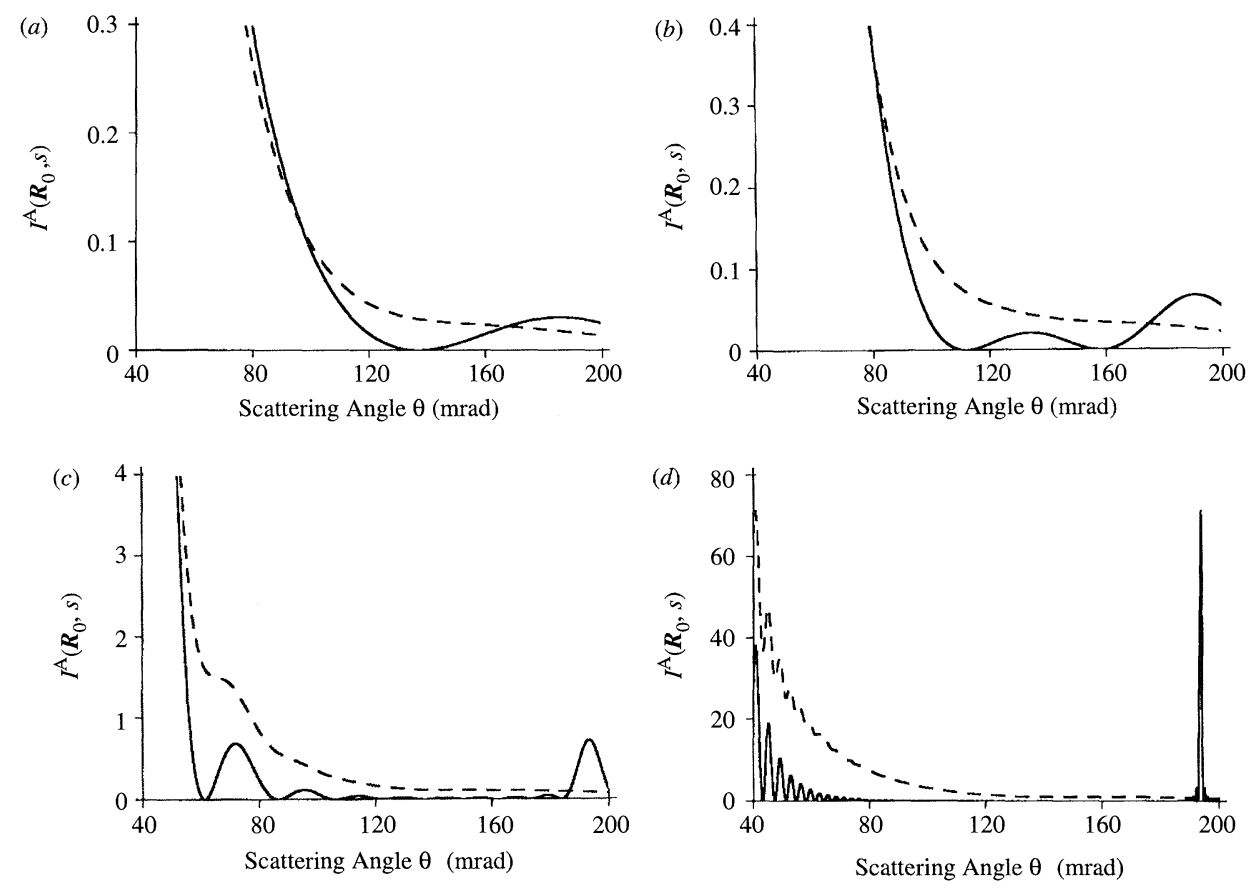

Figure 8. Columnar intensity distributions $I^{\mathrm{A}}\left(\boldsymbol{R}_{0}, s\right)$ (azimuthally integrated) in the detector plane calculated for columns of length (a) $2 \AA,(b) 4 \AA,(c) 18 \AA$, and $(d) 200 \AA$. The dashed line is calculated using the integrand of (5.1) with a Debye-Waller parameter $B=0.45$. This is compared with the coherent calculation (solid line) for stationary atoms $(B=0)$ in all cases. The atom separation along the column is $2 \AA$.

identical, non-vibrating column $(B=0)$. It is evident that for very thin crystal slabs and detector angles considerably smaller than $50 \mathrm{mrad}$, the scattering to the ADF detector can be regarded as perfectly coherent. This is consistent with the stationary atom description of STEM ADF imaging of very thin specimens utilized by Jesson \& Pennycook (1993). However, for the moderately large scattering angles or thicker specimens generally associated with Z-contrast imaging, thermal vibrations play a strong and usually dominant role.

As with our discussion of lateral coherence, we will begin by considering the scattering from a non-vibrating atomic column. In this case, we find that stationary atoms do not give a physically realistic description of the intensity distribution from a column at high angles. However, they do highlight the nature of the coherent effects to be expected and demonstrate that the geometrical role of the ADF detector is far less important than in the case of lateral coherence. In addition a non-vibrating column illustrates the very different signal characteristics associated with zero layer scattering and diffraction to higher-order Laue zones (HOLZs).

\section{(a) Stationary atoms}

In figure $9 a$, we show the thickness dependence of a Z-contrast image of a non-vibrating atomic column. The dramatic departure from the linear thickness dependence of ideal incoherent imaging theory emphasizes the importance of longitudinal coherence in scattering to the high-angle detector. A very short column behaves as a two-dimensional phase object, with the atoms all scattering in phase 

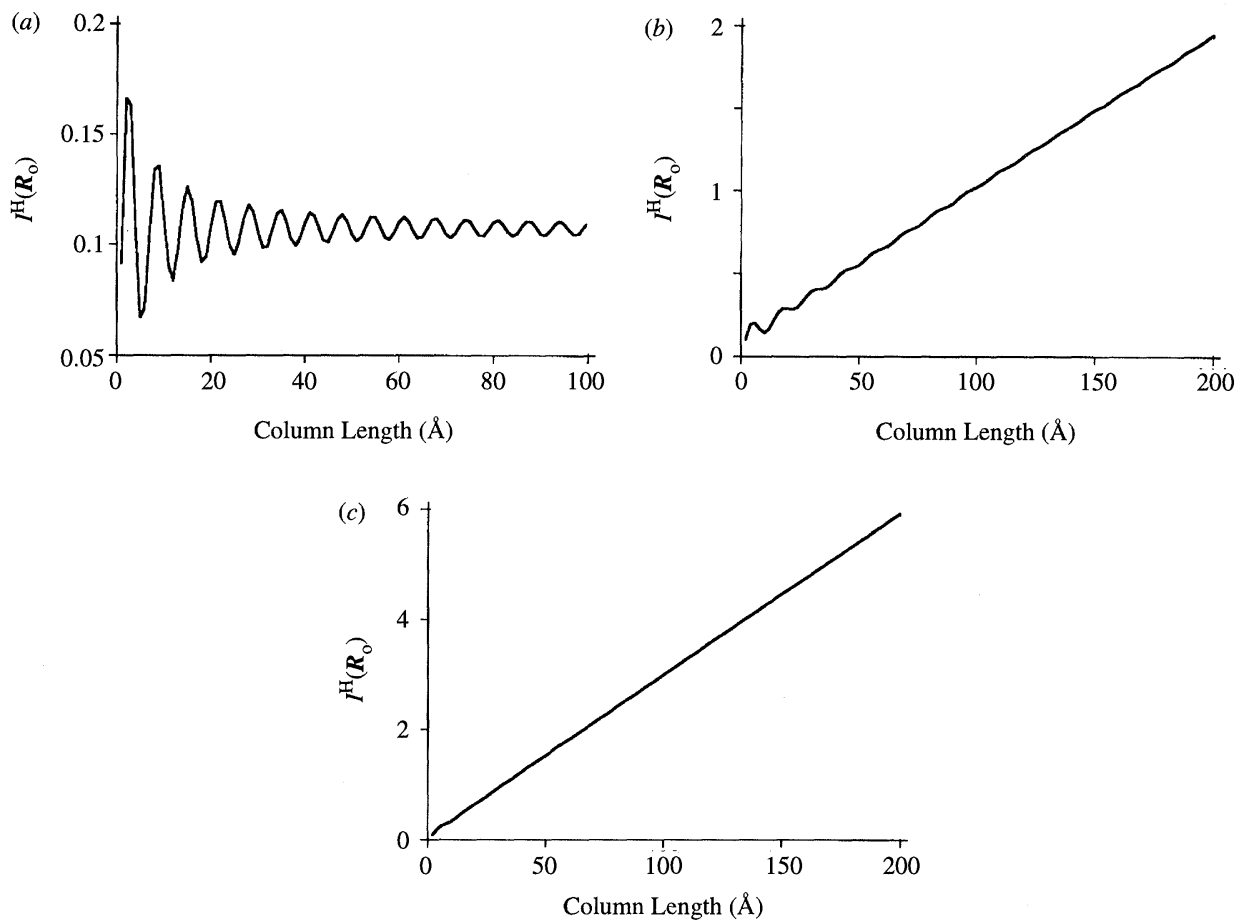

Figure 9. The thickness dependence of a non-vibrating atomic column $(B=0)$ containing atoms spaced $2 \AA$ apart for an (a) 75-150 mrad and (b) 75-200 mrad ADF detector range. In (c), thermal vibrations are added $(B=0.45)$ for the $75-150 \mathrm{mrad}$ range for direct comparison with $(a)$.

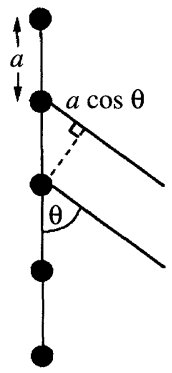

Figure 10. Geometry for scattering to the $n$th columnar HOLZ ring.

to high angles. The intensity, therefore, increases as the number of atoms in the column squared (Jesson \& Pennycook 1993). However, for an inner detector angle of $75 \mathrm{mrad}$, destructive interference is significant for columns only a few $\AA$ in length. Hence, for this detector geometry (which does not include any HoLz lines), the coherent signal from a column never rises above the value associated with the thin crystal phase object, and demonstrates the relative inefficiency of the high-angle detector in destroying longitudinal coherence.

A further illustration of the departure from incoherent imaging theory is apparent in the sensitivity of the signal to detector geometry. Consider, for example, extending the outer angle of the high-angle detector from 150 to $200 \mathrm{mrad}$. This enables the first high-order Laue zone (HOLZ) ring to be detected (see figure 8) and dramatically changes the thickness dependence of the column, as shown in figure $9 b$. HOLZ diffraction is connected with three-dimensional Bragg scatter- 
286

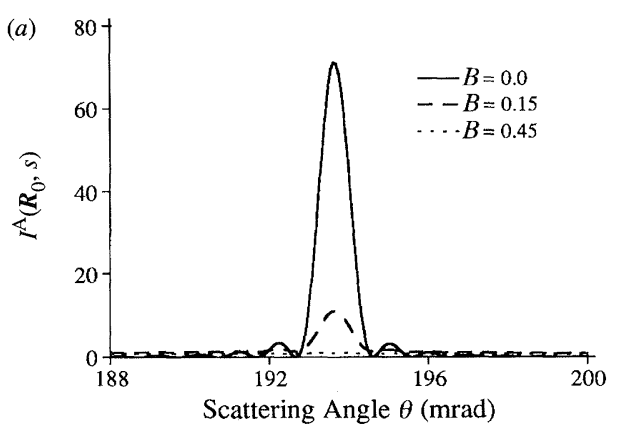

D. E. Jesson and S. J. Pennycook

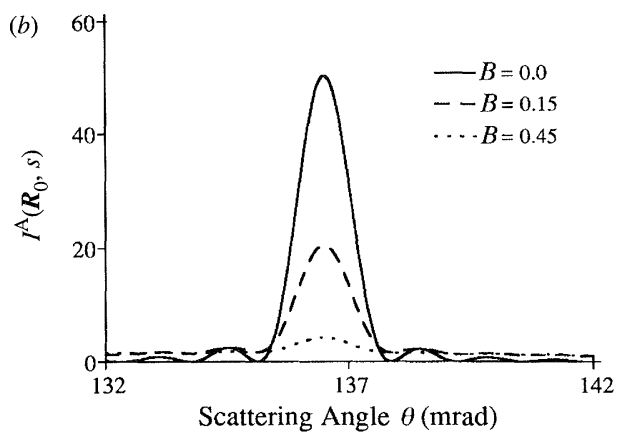

Figure 11. Comparison of HOLZ ring intensities $I^{\mathrm{A}}$ (azimuthally integrated) calculated using the integrand of (5.1) for stationary atoms, $B=0$ (solid line), atoms at room temperature, $B=0.45$ (dotted line), and atoms at absolute zero, $B=0.15$ (dashed line). The atoms are spaced $(a) 2 \AA$ and (b) $4 \AA$ apart along a column of length $200 \AA$.

ing and a simple geometrical argument (figure 10) gives the scattering angle for diffraction to the $n$th columnar HOLZ ring as

$$
\theta_{\mathrm{H}}^{n}=\arccos (1-n \lambda / a),
$$

where $a$ is the spacing along the column. For $a=2 \AA$, we find $\theta_{\mathrm{H}}^{1}=193 \mathrm{mrad}$, which is in excellent agreement with the HOLZ ring position in figure 8 .

The large scattering angles involved render HOLZ diffraction very sensitive to small atomic displacements. As seen in figure 8, the presence of thermal vibrations markedly reduces the strength of HOLz rings. It is important to realize that this is a significant effect even at absolute zero due to zero-point vibrations. For $\mathrm{Si}$ at $0 \mathrm{~K}, B=0.15$ (International tables for $X$-ray crystallography 1962), which still results in a significantly reduced HOLZ intensity compared to the stationary atom prediction, even for the favourable case involving a $4 \AA$ atom spacing along the column (figure 11).

Nevertheless, utilizing a cooled specimen and a thin annular detector, our calculations indicate that a HOLZ ring image might be formed under favourable circumstances which approaches $30 \%$ of the typical signal associated with a Zcontrast image. We, therefore, briefly speculate on the properties and potential uses of such an image. It is well appreciated that HOLZ diffraction is sensitive to the arrangement of atoms along a column in the form of conditional projected potentials (Baker 1983; Vincent et al. 1984; Jesson \& Steeds 1990). For example, two identical atoms in a column separated by half the unit cell repeat distance will cancel completely from the first-order Laue zone intensity. This suggests that a HOLZ ring image could, in principle, provide important three-dimensional information concerning the nature of multicomponent strings in a crystal projection and would be highly complementary to Z-contrast images formed from TDS.

The above discussion clearly demonstrates that HOLZ and TDS contrast mechanisms are fundamentally different. Even in the case of monatomic strings, the discrete nature of HOLZ rings implicates a specific value of the atom scattering factor. In the case of TDS, however, $f^{2}(s)$ is integrated over the entire range of the ADF detector, producing an apparent difference in scattering cross section per atom, as evidenced by the differing slopes in figure 12 . The linear thickness dependence displayed by both HOLZ and TDS has led to understandable confusion in the literature as to the important contrast mechanisms involved in Z-contrast 


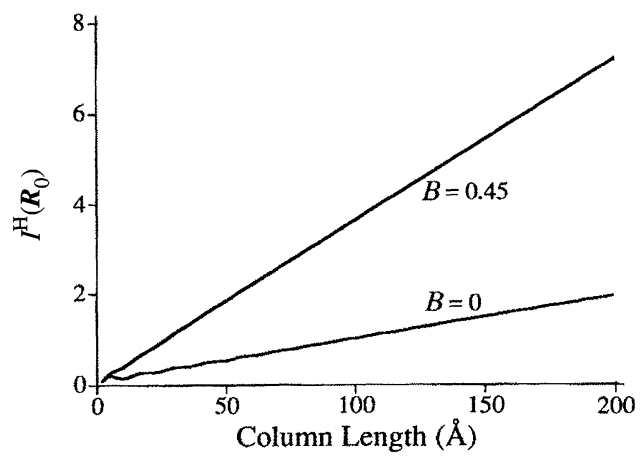

Figure 12. A comparison of the thickness dependence of vibrating $(B=0.45)$ and non-vibrating $(B=0)$ column intensities containing atoms spaced $2 \AA$ apart and a $75-200 \mathrm{mrad}$ ADF detector. The non-vibrating column intensity is dominated by HOLZ diffraction (see figure 8).

imaging (Spence et al. 1989; Loane et al. 1991). It is clear from our calculations that in practice, TDS will dominate in the formation of Z-contrast images and that the resulting contrast is fundamentally different in nature from that to be expected from HOLZ diffraction.

\section{(b) Vibrating atoms}

It is evident from figure 8 that thermal vibrations strongly suppress HOLZ diffraction and modify the scattering distribution in the vicinity of the high-angle detector. In this section, we consider the important role of TDS in suppressing longitudinal coherence. The influence of phonons is described directly by the term,

$$
W_{m n}=\exp \left\{Y_{m n}-2 M\right\}=\exp \left\{2 M\left[\frac{\operatorname{Si}[(m-n) \pi]}{|(m-n) \pi|}-1\right]\right\},
$$

as contained in (5.1). It is convenient to consider $W_{m n}$ as a correlation function which expresses the degree of correlation along a column. For example, by selecting the $m=0$ atom, then $W_{0 n}$ will describe its ability to interfere with the $n$th atom along a column (see figure 13a).

The Einstein model of independent vibrating atoms is the simplest and most commonly used model for TDS. This is equivilent to assuming an Einstein dispersion where the angular frequency $\omega_{q j}$ of all modes and polarizations is equal to a constant value $\omega_{\mathrm{E}}$. In this case, $G_{q j}$ is independent of $q(3.5)$ so that noting $\sum_{q j} G_{q j}=2 M$, we obtain $Y_{m n}=2 M \delta_{m n}$ from (3.7). Equation (5.3) then gives,

$$
W_{0 n}=\exp \left\{2 M\left[\delta_{0 n}-1\right]\right\},
$$

which is a delta function at the zeroth atom and $\exp (-2 M)$ for all other atoms (figure 13c). The zeroth atom is perfectly correlated with itself but sees all other atoms as independent oscillators. For a column of $N_{\mathrm{c}}$ atoms, this very convenient result enables the TDS intensity expression (5.1) to be partitioned into coherent and incoherent terms in the usual way:

$$
\begin{gathered}
I^{\mathrm{H}}\left(\boldsymbol{R}_{0}\right)=\epsilon^{1 \mathrm{~s}^{2}} \tau^{1 \mathrm{~s}^{2}}\left(\boldsymbol{R}_{\mathrm{c}}\right) P^{2}\left(\boldsymbol{R}_{\mathrm{c}}-\boldsymbol{R}_{0}\right)\left[N_{\mathrm{c}} \int_{s_{\mathrm{i}}}^{s_{\mathrm{o}}} f^{2}(s)(1-\exp (-2 M)) 2 \pi s \mathrm{~d} s\right. \\
\left.+\sum_{m, n} \int_{s_{\mathrm{i}}}^{s_{\circ}} f^{2}(s) \exp \left\{\mathrm{i} \frac{s^{2}}{2 \chi} z_{m n}\right\} \exp (-2 M) 2 \pi s \mathrm{~d} s\right] .
\end{gathered}
$$

Proc. R. Soc. Lond. A (1995) 


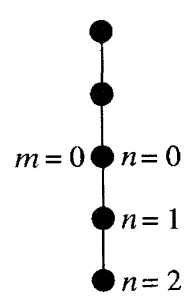

(a)

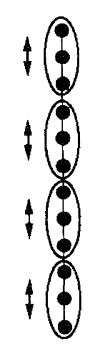

(b)

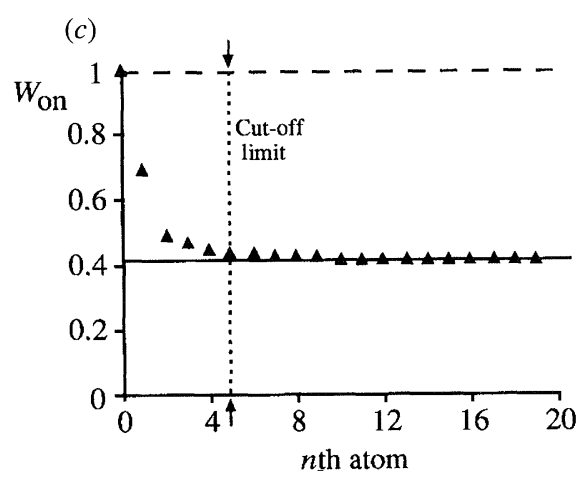

Figure 13. (a) Schematic of an atomic column showing the location of the $n$th atom away from the zeroth atom $(m=0, n=0)$. (b) Representation of the column scattering as individual packets (see text). (c) Values of $W_{0 n}$ in the Warren approximation (triangles), the Einstein limit (solid line) and the coherent limit (dashed line) for $B=0.45$ and $\theta_{\mathrm{i}}=75 \mathrm{mrad}$. The arrowed vertical line defines the packet cut-off limit (see text).

It is important to emphasize, however, that the Einstein model naturally builds incoherence into the scattering process via the use of (5.4). Approaches based on this approximation (see, for example, Loane et al. 1991; Treacy \& Gibson 1993) will, therefore, not correctly describe the coherence length along a column because they do not take into account near-neighbour correlations. The opposite extreme to the Einstein model is obtained by putting $W_{0 n}=1$ for all $n$ (figure $13 c$ ). This corresponds to the stationary atom case discussed in $\S 5 a$ where the zeroth atom is perfectly correlated with all other atoms in the column.

In the Warren approximation, $W_{0 n}$ lies between the Einstein and non-vibrating atom extremes as shown in figure $13 c$. The correlation factor is unity at the origin but falls off rapidly as atoms become less correlated further away from the zeroth atom. Eventually, the curve is asymptotic to the $\exp (-2 M)$ line associated with independent oscillators. This leads to a useful physical interpretation of TDS within the Warren approximation. By defining a packet cut-off limit as shown in figure $13 c$, we can consider the zeroth atom as being partially correlated with atoms contained within the packet and uncorrelated with those atoms outside the packet. It is, therefore, possible to visualize scattering from an atomic column as similar to scattering from an assembly of independent packets of atoms with the important proviso that the atoms contained within a packet are partially coherent.

The division of a column of atoms into packets (figure 13b) enables (5.1) to be partitioned into coherent and incoherent terms in a manner directly analogous to 
the Einstein expression (5.5). For a column of $N_{\mathrm{c}}$ atoms, we can write

$$
\begin{aligned}
I^{\mathrm{H}}\left(\boldsymbol{R}_{0}\right)=\epsilon^{1 \mathrm{~s}^{2}} \tau^{1 \mathrm{~s}^{2}}\left(\boldsymbol{R}_{\mathrm{c}}\right) P^{2}\left(\boldsymbol{R}_{c}-\boldsymbol{R}_{0}\right)\left[N_{\mathrm{p}} \sum_{n} \int_{s_{\mathrm{i}}}^{s_{\mathrm{o}}} f^{2}(\boldsymbol{s}) \cos \left\{\frac{\pi s^{2}}{\chi} z_{0 n}\right\}\right. \\
\times\left[\exp \left\{2 M\left(\frac{\operatorname{Si}(n \pi)}{|n \pi|}-1\right)\right\}-\exp (-2 M)\right] 2 \pi s \mathrm{~d} s \\
\left.+\sum_{m, n} \int_{s_{\mathrm{i}}}^{s_{\mathrm{o}}} f^{2}(\boldsymbol{s}) \exp \left\{\frac{\mathrm{i} s^{2}}{2 \chi} z_{m n}\right\} \exp (-2 M) 2 \pi s \mathrm{~d} s\right] .
\end{aligned}
$$

The second term in this expression is identical to the usual coherent scattering term of the Einstein approximation (5.5). The first term describes incoherent scattering from $N_{\mathrm{p}}$ individual packets (to a good approximation $N_{\mathrm{p}}=N_{\mathrm{c}}$ ) with the summation performed over the contents of a packet. The number of atoms in the packet depends on both the detector inner-angle and Debye-Waller factor, but is independent of atom spacing along a column. Figure 14 illustrates the effect of Debye-Waller factor on packet size and the thickness dependence of the detected signal. Upon decreasing $B$ from 2.0 to 0.4 , it is necessary to increase the packet size from three to at least seven atoms to obtain accurate agreement between (5.6) and the full calculation using (5.1) for $\theta_{\mathrm{i}}=75 \mathrm{mrad}$. In figure 15 , we show a similar relationship between the packet size and the thickness dependence for different values of the inner detector angle $\theta_{\mathrm{i}}$.

An important prediction of the packet approximation is that at sufficiently high angles, where the coherent Bragg scattering term of (5.6) can be neglected, the column intensity will increase linearly with thickness. However, the residual partial coherence evident within each packet (figure 13c) will change the effective scattering cross section compared with the Einstein independent oscillator model (5.5). For example, the scattering to the high-angle detector can be above or below the predictions of the Einstein model depending on the atom spacing along a column (figure $16 a$ and $b$ ), which influences the net amplitude contributed by an individual packet.

It is clear, therefore, that phonons efficiently break the coherence along a column, as is evident from a comparison of the columnar thickness dependencies contained in figure $9 a$ and $c$, but there is still residual partial coherence involving atoms contained within a packet. The outstanding question, therefore, concerns the practical significance of these effects when using an Einstein model to quantify column intensities. Consider, for example, two columns both of length $80 \AA$, but with different atomic separations of $1 \AA$ and $2 \AA$ along the column. Calculations show that the use of the Einstein model can underestimate the column intensity ratio by $57 \%$. This is a rather extreme example since in practice spacings as small as $1 \AA$ are not generally encountered. A more typical figure is a $16 \%$ underestimate associated with two $80 \AA$ columns involving spacings of 2 and $4 \AA$. Therefore, although packets containing several atoms can contribute to longitudinal coherence in Z-contrast images, the coherence within a packet is only partial, which limits these effects to a relatively small departure from the independent oscillator models for atom spacings usually encountered in practice.

\section{Discussion}

In this paper, we have utilized the Warren approximation to bridge the gap between a full phonon and independent oscillator treatment of TDS. The Debye 

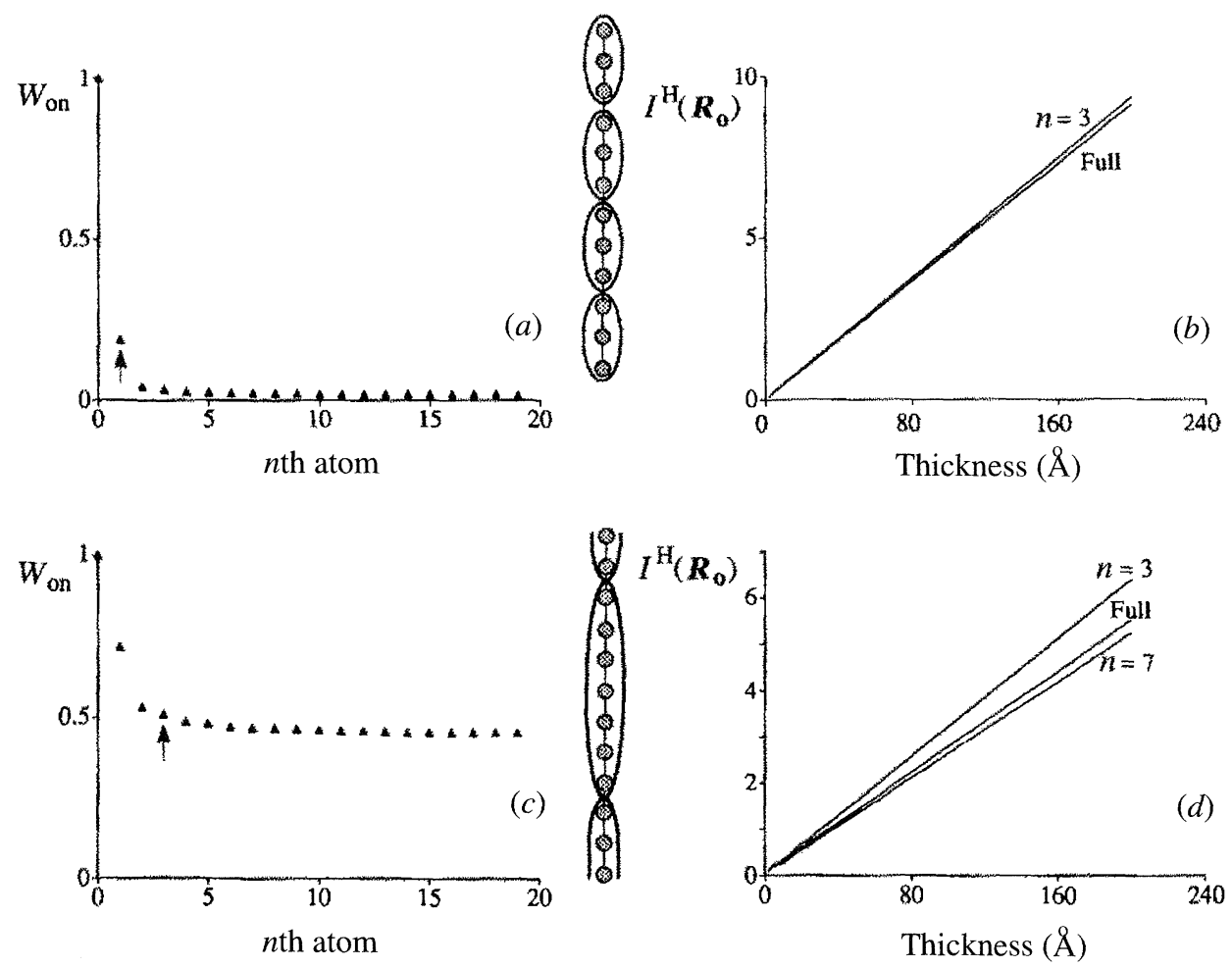

Figure 14. (a) Correlation function $W_{0 n}$ for $\theta_{\mathrm{i}}=75 \mathrm{mrad}$ and $B=2.0$. The arrow defines the packet cut-off limit. (b) Columnar thickness dependence calculated using the full expression (5.1) and the packet approximation (5.6) for a 75-150 mrad ADF detector $(B=2.0)$. A packet consisting of three atoms is sufficient to obtain good convergence. $(c)$ and $(d)$ correspond to $(a)$ and $(b)$, respectively, but with $B=0.4$, which requires a seven-atom packet for convergence. The atom spacing along the column is $2 \AA$.

model used probably tends to somewhat overestimate atomic correlations by assigning excessively high frequencies (and hence low vibration amplitudes) to the shortest wavelength modes (Anderson et al. 1982; Nielsen \& Weber 1980). It should, however, provide a useful means of assessing the limitations of the Einstein model of thermal vibrations as a basis for quantifying Z-contrast images. It is apparent from the analysis that atoms located in different atomic columns of a crystal projection can be considered to scatter independently. However, atomic columns scatter as an assembly of independent packets of atoms in which the atoms contained within a packet are partially coherent. This means that the intensity of a column can be above or below the Einstein model prediction depending on the phase of the residual partial coherence.

It should be emphasized that partial coherence along a column is generally neglected in most TDS calculations based on the multislice approach (see, for example, Wang \& Cowley 1989). Often, it is assumed that individual slices scatter incoherently even though coherence effects on the scale of a packet dimension are evident in figure 7 of Doyle (1969). Similarly, each slice of a crystal is generally considered to scatter incoherently in calculations based on the Bloch wave formalism (Young \& Rez 1975; Rez et al. 1977). The justification for this is usually based on the assumption that matrix elements governing transitions between 

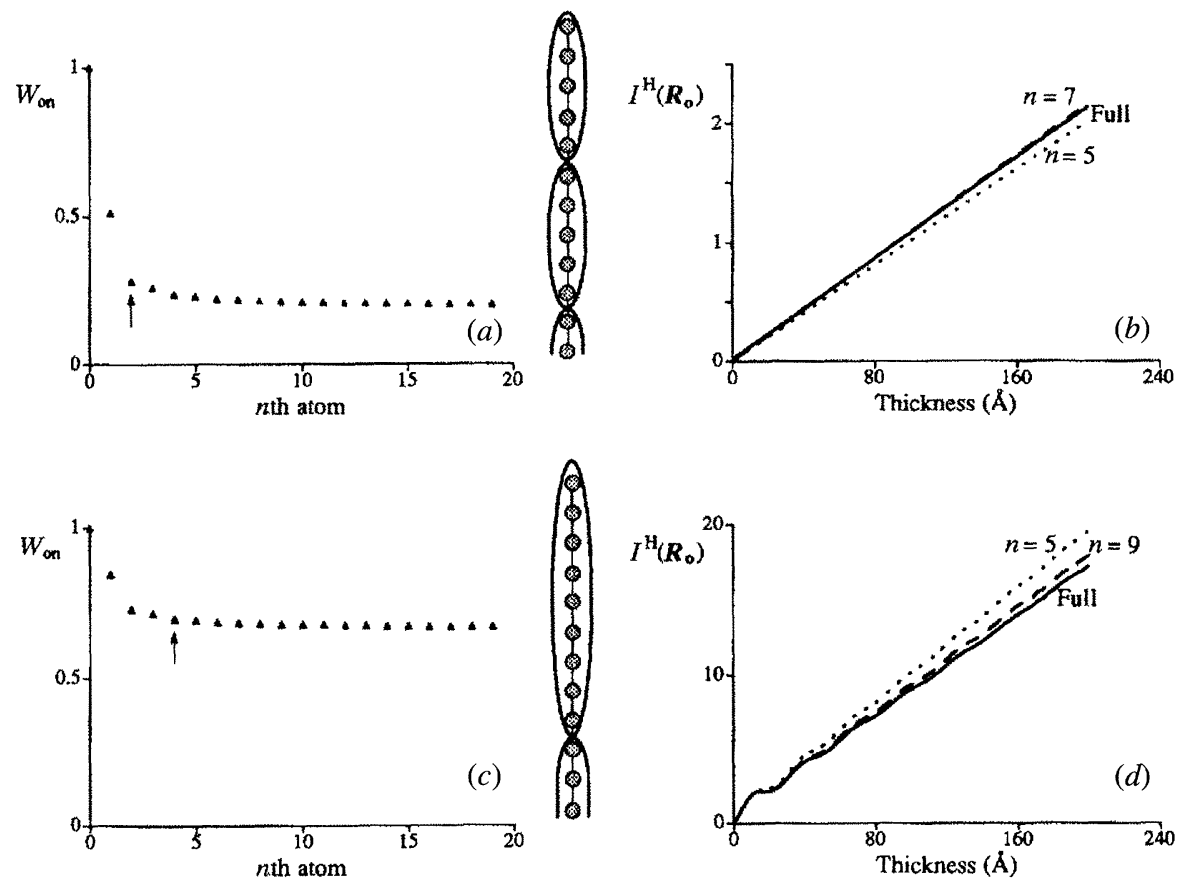

Figure 15. (a) Correlation function $W_{0 n}$ for $\theta_{\mathrm{i}}=100 \mathrm{mrad}$ and $B=0.45$. The arrow defines the packet cut-off limit. (b) Columnar thickness dependence calculated using the full expression (5.1) and the packet approximation (5.6) for a 100-150 mrad ADF detector and $B=0.45$. A packet consisting of five atoms is sufficient to obtain accurate convergence. $(c)$ and $(d)$, respectively, correspond to $(a)$ and $(b)$ but with $\theta_{\mathrm{i}}=50 \mathrm{mrad}$ in $(c)$ and a detector range of 50-150 mrad in $(d)$. A packet consisting of nine atoms is required for accurate convergence. The atom spacing along the column is $23 \AA$.

branches of the dispersion surface vary slowly with the $z$ component of the phonon wavevector equal to $2 \pi n / t$, where $t$ is the crystal thickness. It is then possible to take the matrix elements outside of the summation,

$$
\sum_{n} \exp \left\{\frac{2 \pi \mathrm{i} n}{t}\left(z-z^{\prime}\right)\right\}
$$

to obtain the independent olice result $N_{\mathrm{c}} \delta\left(z-z^{\prime}\right)$. This approximation is, however, least valid for near-neighbour correlations (i.e. small $\left(z-z^{\prime}\right)$ ) within a packet.

The dominant influence of packets in the context of Z-contrast imaging is to modify the effective scattering cross section per atom. Partial coherence limits this effect to typically less than $20 \%$ for most cases of practical importance. For atomic strings of length $t$ containing a single atom type, the appropriate intensity expression, is by analogy with the result of Pennycook \& Jesson (1991);

$$
I^{\mathrm{H}}\left(\boldsymbol{R}_{0}, t\right)=O\left(\boldsymbol{R}_{0}, t\right) * P^{2}\left(\boldsymbol{R}_{0}\right)
$$

where $O\left(\boldsymbol{R}_{0}, t\right)$ is the object function given by

$$
O\left(\boldsymbol{R}_{i}, t\right)=\sum_{i} \sigma_{i}^{\mathrm{c}} \epsilon^{\mathrm{ls}^{2}} \tau^{\mathrm{ls}^{2}}\left(\boldsymbol{R}_{i}, 0\right)\left[\frac{1-\exp \left(-2 \mu^{\mathrm{ls}} t\right)}{2 \mu^{\mathrm{ls}}}\right] .
$$

Proc. R. Soc. Lond. A (1995) 

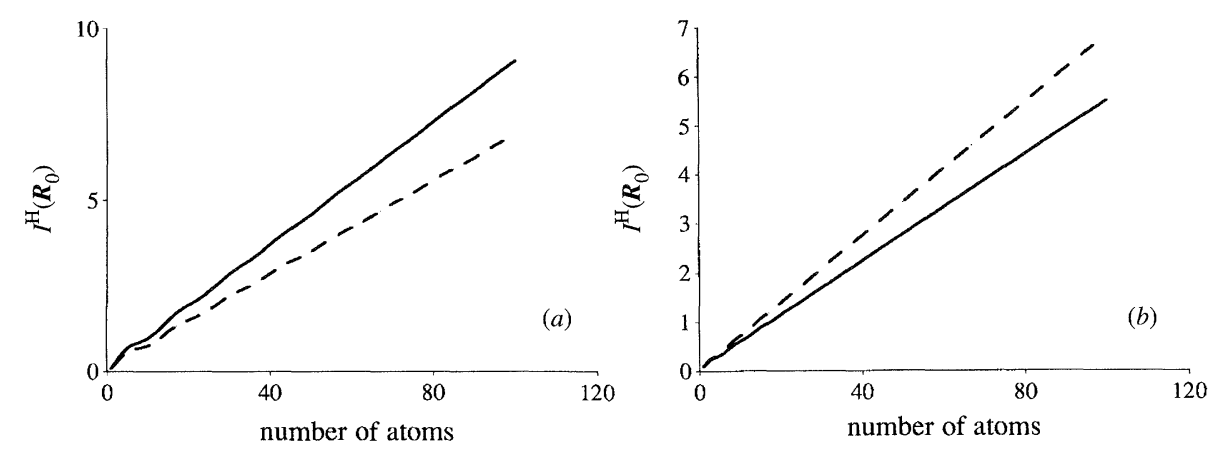

Figure 16. Columnar intensity thickness dependence calculated in the Einstein (dashed line) and Warren (solid line) approximations for atoms spaced at (a) $1 \AA$ and (b) $2 \AA$ along the column. The detector range is $75-150 \mathrm{mrad}$ and $B=0.45$.

Here $\mu^{\text {ls }}$ is the axial s-state absorption. Since the packet size is considerably smaller than the absorption length, $\sigma_{i}^{\mathrm{c}}$ can be calculated as before. For a column of $N_{\mathrm{c}}$ atoms,

$$
\begin{aligned}
\sigma_{i}^{\mathrm{c}}=N_{\mathrm{c}} & \sum_{n} \int_{s_{\mathrm{i}}}^{s_{\mathrm{o}}} f^{2}(s) \cos \left\{\frac{\pi s^{2}}{\chi} z_{0 n}\right\} \\
\times & {\left[\exp \left\{2 M\left[\frac{\operatorname{Si}(n \pi)}{|n \pi|}-1\right]\right\}-\exp (-2 M)\right] 2 \pi s \mathrm{~d} s . }
\end{aligned}
$$

The effective probe profile is given by

$$
P^{2}\left(\boldsymbol{R}_{0}\right)=\left|\frac{1}{\epsilon^{\mathrm{ls}}(0)} \int_{\text {probe }} \epsilon^{\mathrm{ls}}(\boldsymbol{K}) \operatorname{expi}\left[\boldsymbol{K} \cdot\left(\boldsymbol{R}-\boldsymbol{R}_{0}\right)+\gamma(\boldsymbol{K})\right] \mathrm{d} \boldsymbol{K}\right|^{2},
$$

where $\gamma(\boldsymbol{K})$ is the usual phase factor due to spherical abberation and defocus. Note that atomic correlations in TDS must also influence the Z-contrast image intensity via the attenuation of s-states. It is possible to include these effects approximately through the derivation of an absorption potential based on the Warren treatment of atomic correlations (Jesson \& Pennycook 1994, unpublished work). Absorption coefficients calculated from this potential can then be included explicitly in equation (6.2). The case of multicomponent atomic strings involves a more complicated scaling of the columnar scattering cross section and will be considered in detail in a future publication.

\section{Conclusions}

We have investigated large-angle electron scattering in the framework of the Warren treatment of thermal vibrations. It is shown that lateral coherence involving scattering between atoms located in different atomic columns is efficiently removed in Z-contrast imaging as a result of the high-angle detector geometry. Longitudinal coherence involving atoms constrained within the same column is also largely suppressed as a result of thermal vibrations. However, residual longitudinal coherence does remain and can be visualized as partially coherent scattering from an assembly of independent packets. Under typical conditions, this can modify the columnar intensity by around $20 \%$, somewhat justifying the use of an independent oscillator model. 
We thank S. L. Carney, T. C. Estes, and J. T. Luck for technical assistance. This work was sponsored by the Division of Materials Sciences, U.S. Department of Energy, under contract DE-AC05-84OR21400 with Martin Marietta Energy Systems, Inc.

\section{References}

Ade, G. 1977 Optik 49, 113-116.

Anderson, J. U., Bonderup, E., Laegsaard, E., Marsh, B. B. \& Sørensen, A. H. 1982 Nucl. Instrum. Methods 194, 209-224.

Baker, J. R. 1983 Structure information from higher-order Laue zone line intensities in high energy electron diffraction. Ph.D. thesis, University of Bristol, U.K.

Colliex, C. \& Mory, C. 1983 In Quantitative electron microscopy (ed. J. N. Chapman \& A. J. Craven), pp. 149-216. SUSSP.

Cowley, J. M. 1976 Ultramicroscopy 2, 3-16.

Cowley, J. M. \& Moodie, A. F. 1962 J. Phys. Soc. Jap. 17 (Suppl. BII), 86.

Crewe, A. V. 1970 Q. Rev. Biophys. 3, 137-179.

Crewe, A. V. \& Wall, J. 1970 Optik 30, 461-474.

Doyle, P. A. 1969 Acta crystallogr. A 25, 569-577.

Fertig, J. \& Rose, H. 1981 Optik 59, 407-429.

Gibson, J. M. \& Howie, A. 1978-79 Chem. Scr. 14, 109-116.

Hall, C. R. 1965 Phil. Mag. 12, 815-826.

Howie, A. 1979 J. Micros. 117, 11-23.

Isaacson, M., Ohtsuki, M. \& Utlaut, M. 1979 Introduction to analytical electron microscopy (ed. J. J. Hren, J. Goldstein \& D. C. Joy), pp. 343-368. New York and London: Plenum Press.

James, R. W. 1954 The optical principles of the diffraction of X-rays, pp. 193-267. London: G. Bell and Sons.

Jesson, D. E. \& Steeds, J. W. 1990 Phil. Mag. A 61, 363-384.

Jesson, D. E. \& Pennycook, S. J. 1993 Proc. R. Soc. Lond. A 441, 261-281.

Loane, R. F., Kirkland, E. J. \& Silcox, J. 1988 Acta crystallogr. A 44, 912-927.

Loane, R. F., Xu, P. \& Silcox, J. 1991 Acta crystallogr. A 47, 267-278.

Nielsen, O. H. \& Weber, W. 1980 J. Phys. C 13, 2449-2460.

Pennycook, S. J. \& Jesson, D. E. 1990 Phys. Rev. Lett. 64, 938-941.

Pennycook S. J. \& Jesson, D. E. 1991 Ultramicroscopy 37, 14-38.

Pennycook, S. J. \& Jesson, D. E. 1992 Acta metall. mater. 40, S149-S159.

Rez, P., Humphreys, C. J. \& Whelan, M. J. 1977 Phil. Mag. 35, 81-96.

Schiff, L. I. 1968 Quantum mechanics, 3rd edn, p. 327. New York: McGraw-Hill.

Spence, J. C. H., Zuo, J. M. \& Lynch, J. 1989 Ultramicroscopy 31, 233-240.

Takagi, S. 1958 J. Phys. Soc. Jap. 13, 278-286.

Treacy, M. M. J. \& Gibson J. M. 1993 Ultramicroscopy 52, 31-53.

Vincent R., Bird, D. M. \& Steeds, J. W. 1984 Phil. Mag. A 50, 765-786.

Wall, J., Langmore, J., Isaacson, M. \& Crewe, A. V. 1974 Proc. natn. Acad. Sci. USA 71, 1-5.

Wang, Z. L. \& Cowley, J. M. 1989 Ultramicroscopy 31, 437-453.

Wang, Z. L. \& Cowley, J. M. 1990 Ultramicroscopy 32, 275-289.

Warren, B. E. 1990 X-ray diffraction. New York: Dover.

Young, A. P. \& Rez, P. 1975 J. Phys. C 8, L1-L7.

International Tables for X-Ray Crystallography 1962 Vol. III, p. 234. Birmingham, U.K.: Kynoch Press. 


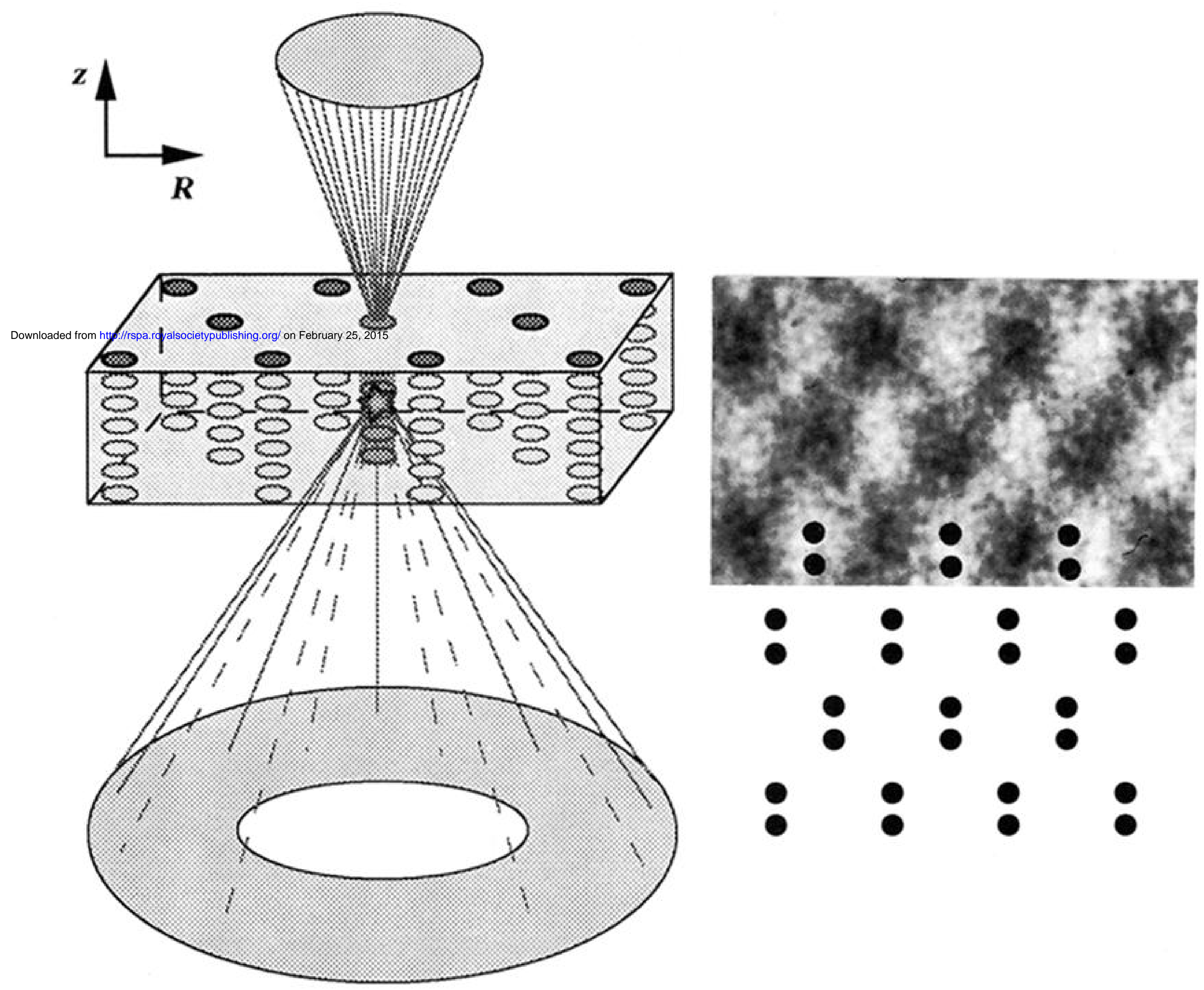

Figure 1. A schematic illustration of the Z-contrast imaging geometry. A focused electron probe channels along the projected atom columns and undergoes a large-angle scattering event to the high-angle detector. Images are formed by scanning the electron probe across the surface and can be interpreted in terms of the projected specimen scattering power. The $\operatorname{Si}\langle 110\rangle$ example at $100 \mathrm{kV}$ illustrates a case where the individual atomic columns comprising a dumbbell separated by $1.36 \AA$ cannot be resolved by the $2.2 \AA$ FWHM probe. 

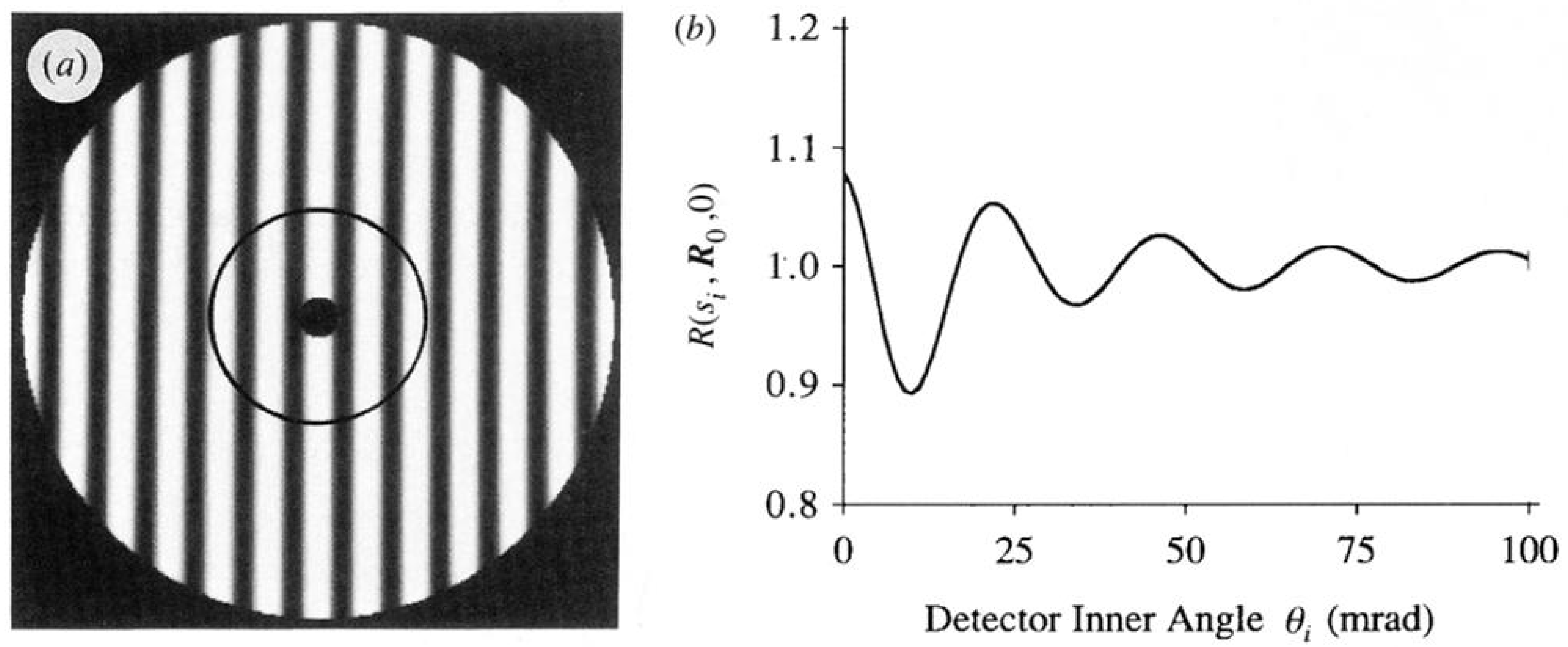

Figure 4. (a) Detector plane intensity distribution resulting from two point scatterers separated by $1.5 \AA$. The inner and outer detector angles are 10.3 and $150 \mathrm{mrad}$, respectively. The solid circle shows that by increasing the inner detector angle to $50 \mathrm{mrad}$, more interference fringes are sampled at the detector periphery. (b) Ratio of the ADF signal to the incoherent signal $\left[\boldsymbol{R}\left(s_{i}, \boldsymbol{R}_{0}, 0\right)\right]$ for two stationary $\mathrm{Si}$ atoms separated by $1.5 \AA$ as a function of inner detector angle. The probe is located between the atom pair. 